\title{
Verändert die Marktmiete die residenzielle Segregation? Die Marktmiete aus sozial- und wirtschaftsgeographischer Sicht
}

\section{Joris Ernest Van Wezemael, André Odermatt, Zürich}

Menschen machen nicht nur fortwährend ihre eigene Geschichte, sondern auch ihre eigene Geographie. Sie machen diese nicht aus freien Stücken, nicht unter selbst gewählten Umständen. Eine Aufgabe der Sozialund Wirtschaftsgeographie besteht darin, das Handeln und die Handlungsfolgen jener Aktoren zu untersuchen, welche aufgrund ihres grossen Handlungspotenzials in bedeutendem Masse Geographie machen. Weil die ( $\mathrm{Re}$-)Produktion einer bestimmten räumlichen und sozialen Ordnung in aller Regel nicht das eigentliche Ziel des Handelns darstellt, gilt das Interesse sozial- und wirtschaftsgeographischer Untersuchungen besonders den unbeabsichtigten Folgen zweckgerichteten Handelns. Der vorliegende Beitrag diskutiert am Beispiel der Schweiz, wie aufgrund der vorerst noch hypothetischen Einführung der Marktmiete die räumlich-soziale Wohnstandortverteilung (residenzielle Segregation) verändert würde. Dabei steht die These im Zentrum, wonach die räumlichsoziale Wohnstandortverteilung ein Kernelement der Handlungsorientierung der Aktoren innerhalb der sozioökonomischen Institution Wohnungsimmobilienmarkt darstellt.

\section{Die Renaissance des Marktes}

Staatliche Eingriffe in das Marktgeschehen sind umstritten. Gegenwärtig ist ein eigentlicher Paradigmawechsel im Hinblick auf die Rolle des Staates im Gang. Die Nachkriegsära war in den westlichen Zentrumsgesellschaften (BORNSCHIER 1998), also auch in der Schweiz, geprägt durch das Produktionsparadigma der Massenproduktion fordistisch-tayloristischer Prägung und einen Staat, der den aufgrund steigender wirtschaftlicher Leistung wachsenden Reichtum umverteilte. So verhalf er einem breit abgestützten Bild sozialer Gerechtigkeit zur realen Existenz. Seit den 70er Jahren wurden aber Risse im Tandem des Staates keynesianischer Prägung und der Massenindustrie sichtbar, welche sich durch wiederkehrende wirtschaftliche Krisen bemerkbar machten. Die seit Mitte der 80er Jahre aufgeflammte Deregulierungsdebatte zeichnet sich durch eine Renaissance des Glaubens an den Markt aus (für die Schweiz siehe De Pury et al. 1995). Alles, was heutzutage dereguliert wird, wurde früher einmal reguliert. Dies weist darauf hin, dass Gedanken über mögliche unbeabsichtigte Folgen von Marktlibe- ralisierungen von grosser Bedeutung sind, damit nicht einer Modewelle gleich einem Paradigma (und damit einer Wahrheit auf Zeit) gefolgt wird, ohne dass die Folgen genügend überblickt werden.

\section{Die Marktmiete-Debatte in Politik und Wissenschaft}

Die politische Diskussion um die Einführung der Marktmiete steckt seit geraumer Zeit in einer PattSituation (Van Wezemael 1999: 104). Die Uneinigkeit geht so weit, dass Befürworter und Gegner die Begriffe Kosten- bzw. Marktmiete nicht einmal einheitlich verwenden. Im vorliegenden Beitrag gelten folgende Definitionen:

- Die Marktmiete ist der Preis für die Nutzung von Wohnraum, welcher frei von staatlichen Regulierungen allein durch das Verhältnis von Angebot und Nachfrage bestimmt wird (BUNDESAMT FÜR WOHNUNGSWESEN 1993: 26).

- Die Kostenmiete beruht auf dem Grundsatz, dass eine Miete in erster Linie kostendeckend sein muss. Zur Kalkulation gibt es anerkannte betriebswirtschaftliche Grundsätze (FARAGo et al. 1993: 49).

Das Mietrecht in der Schweiz bildet einen Mittelweg zwischen Kosten- und Marktmiete (BUNDESAMT FüR WOHNUNGSWESEN 1993: 40-41). Während die Marktmiete für Mietervertreter einen Irrweg darstellt, ist sie für die vornehmlich politisch liberalen Befürworter eines Systemwechsels das Fernziel ihrer Mietpolitik (FDP 2000). Die anfangs der 90er Jahre vom Vorsteher des Volkswirtschaftsdepartements eingesetzte Arbeitsgruppe Ordnungspolitik empfahl die Liberalisierung zahlreicher Märkte, u.a. auch des Mietwohnungsmarktes. In der Folge wurde die Expertenkommission Marktmiete (BUNDESAMT FÜR WOHNUNGSWESEN 1993) beauftragt, die Wünschbarkeit der Marktmiete zu erörtern. Ein Konsens wurde nicht erzielt Im Gegensatz zu anderen wichtigen Märkten (z.B. jenem für Telekommunikation) kommt die Deregulierung des Mietwohnungsmarktes nicht voran. Die bisherige Forschung zur Marktmiete in der Schweiz besteht im wesentlichen aus zwei Auftragsstudien der Hochschule St. Gallen (SCHIPS \& MÜLLER 1993, JäGER \& BüHLER 1995). Aus einer sozial- und wirtschaftsgeographischen Perspektive ist das Thema Marktmiete aber nicht hinreichend mit («mainstream-») ökonomischen Ansätzen (Smelser \& Swedberg 1994: 3-4) zu erfassen. Als bedeutendes Defizit der bisherigen Ansätze erscheint der Ausschluss der räumlichen Dimension. Dies ist 
deshalb gravierend, weil die (relative) Lage von Wohnungen eine der wichtigsten Bestimmungsgrössen für deren Marktwert ist («Asset Location») und der räumliche Niederschlag von Investitionsentscheiden die gebaute Umwelt und somit unsere Siedlungen konstituiert. Zudem wird die Wohn- und Lebensqualität sowie die Begehrtheit von Quartieren in erster Linie über räumliche Nähe bzw. Ferne zu erwünschten bzw. unerwünschten Dingen und Personen vermittelt. Eine kritische Würdigung des Forschungsstandes findet sich in VAN WeZemael (1999: 106-118).

Die Untersuchung der Grenze der Bewusstheit und der unbeabsichtigten Folgen für die Systemreproduktion betrachtet GiDDENS (1995: 336) als eine der wichtigsten Aufgaben der Sozialwissenschaften. Neben dem Unbewussten gibt es stets unerkannte Handlungsbedingungen sowie unbeabsichtigte und oftmals nicht wahrgenommene Folgen. Diese Handlungsfolgen werden zu Bedingungen weiteren Handelns. Die Zielorientierung bzw. die Intentionalität der Aktoren ist somit für das Verständnis sozialer Wirklichkeit von grosser Bedeutung, denn die Untersuchung der subjektiv verfolgten Ziele erlaubt es erst, beabsichtigte von unbeabsichtigten Handlungsfolgen zu trennen. Beispielsweise ist es für «Segregationsprozesse» massgeblich, dass die soziale Bedeutung von Objekten durch verschiedene Aktoren einheitlich dechiffriert, aber ihre Bedeutung selbst unterschiedlich bewertet wird. Um dies untersuchen zu können, ist die Zielorientierung der Handelnden zwingend in die Untersuchung einzubeziehen. Entsprechend der genannten Zielsetzung der Sozialwissenschaften steht die räumlich-soziale Wohnstandortverteilung als Nebenergebnis von Wohnbauinvestitionen im Zentrum der weiteren Ausführungen. Es stellt sich also die Frage, ob bzw. wie sich die Liberalisierung des Mietwohnungsmarktes auf die Reproduktion der räumlichsozialen Wohnstandortverteilung auswirken könnte. Der Begriff der räumlich-sozialen Wohnstandortverteilung bezieht sich auf die ungleiche Verteilung von (sozialen) Bevölkerungsgruppen im Hinblick auf räumliche Einheiten eines Gebietes (z.B. einer Stadt) und wird sowohl als Zustand wie auch als Prozess verstanden (LICHTENBERGER 1998: 241).

\section{Der Beitrag der Sozial- und Wirtschaftsgeographie}

Wohnungen stehen in einem physisch-räumlichen und sozialen Kontext (Bourdieu 1991:25). Aus der Verbindung von Wohnungen zu anderen Wohnungen resultieren siedlungsstrukturelle Zusammenhänge. Die Siedlungen in ihren konkret-historischen Ausformungen als Bestandteil der Kulturlandschaft sind in der sozialgeographischen Tradition HARTKEs als «das Nebenergebnis menschlichen Lebens und Handelns» (HARTKE 1959: 426) zu verstehen. So ist die räumlich-soziale
Wohnstandortverteilung in erster Linie als eine unbeabsichtigte Folge zweckgerichteter Handlungen zu verstehen. Die bestehenden Siedlungsstrukturen als situative Rahmenbedingung bilden ein wichtiges Kriterium für zukünftige Entscheidungen, wo investiert werden soll, denn die zum Zeitpunkt der Investitionsentscheidung manifeste soziale Prägung eines Quartiers fördert bestimmte Investitionsarten und beschränkt andere. Weil bestimmte Gebäudetypen als Elemente der Siedlungsstruktur tendenziell mit bestimmten sozialen Bewohnerkategorien korrespondieren (ODERMATT 1997: 202; 303-307), führt eine Veränderung der Baustruktur zu einer veränderten sozialen Zusammensetzung der Wohnbevölkerung. Die räumlich-soziale Wohnstandortverteilung wird unter der Verwendung der Terminologie von Giddens (1995) zu einer Struktur in Form von Erinnerungsspuren, auf welche sich die Aktoren in ihrem Handeln beziehen. Die räumlichsoziale Wohnstandortverteilung wird im Handeln der Aktoren exemplifiziert bzw. reproduziert oder transformiert. Die residenzielle Segregation ist also zugleich eine Folge von Handlungen (Investitionsentscheide, Praxis des Unterhalts und der Vermietung) als auch eine Bedingung für weitere Handlungen. Sie ist folglich weder ein starrer Zustand, noch ist sie frei variabel. Sie ist veränderlich und trotzdem erstaunlich persistent. Historische Stadtbeschreibungen (z.B. BäRTSCHI 1983 ) illustrieren dies auf eindrückliche Weise. Dass die räumlich-soziale Wohnstandortverteilung dennoch zeitlich variiert, weist darauf hin, dass die Prozesse der Reproduktion selber variabel sind.

Je grösser das umgestaltende Vermögen von Handlungen ist, desto weiter reichen ihre Folgen, und zwar in aller Regel die beabsichtigten wie die unbeabsichtigten (GIDDENS 1995: 65-67; 315-320 sowie WERLEN 1997: 304-312). Deshalb ist unser Augenmerk auf jene Aktorkategorien zu richten, die über hohe Vermögensgrade der Transformation bzw. der Reproduktion verfügen, sprich: in bedeutendem Mass Geographie machen. In Bezug auf die räumlich-soziale Wohnstandortverteilung ist es deshalb angezeigt, sich mit Investoren und deren Handeln innerhalb der Sphäre des Wohnungsimmobilienmarktes auseinanderzusetzen. Die Verbindung zwischen den Investoren und den Mietern wird durch die sozioökonomische Institution des Mietwohnungsmarktes hergestellt. Die Mieter sind mittels ihrer Umzüge bzw. der Entscheidung zu bleiben ebenfalls an der Reproduktion der räumlich-sozialen Wohnstandortverteilung beteiligt. Die Geographien der Konsumption (Mieterseite) werden mit der Produktionsseite (Investorenseite) in der Einheit des Produkts «Wohnung» vereint (WERLEN 1997: 313). Analog zur residenziellen Segregation als Handlungsbedingung wirken die spezifischen (rechtlichen) Handlungsbedingungen innerhalb der Sphäre des Wohnungsimmobilienmarktes ermöglichend bzw. einschränkend in bezug auf bestimmte Handlungsweisen. Denn Eigentums- 
und Mietrecht sind zentrale Formen, Verfügungs- und Zugangsbereiche zu regeln (WERLEN 1997: 305).

Aufgrund der bisher entwickelten Argumentation lässt sich folgende These formulieren: Wenn die Marktsphäre mit ihrem unbezweckten Teilergebnis der räumlich-sozialen Wohnstandortverteilung im Zuge einer Liberalisierung des Mietrechts verändert wird, so ist zu erwarten, dass (in erster Linie angebotsseitige) Prozesse der Reproduktion der residenziellen Segregation modifiziert werden. Folglich ist bei der Einführung der Marktmiete mit (unbeabsichtigten) räumlich-sozialen Folgen zu rechnen.

\section{Methodik}

Der hypothetische Charakter der Untersuchung einer möglichen Einführung der Marktmiete legt die Verwendung von Tiefeninterviews (FLICK 1998) nahe. Mittels Experteninterviews wurde im Winter 1999 mit Vertretern der wichtigsten Investorengruppen die Frage erörtert, welche Bedeutung das Räumliche für die Konstitution und Reproduktion sozialer Wirklichkeit hat. Konkret interessierte der Stellenwert der räumlichsozialen Wohnstandortverteilung (bzw. die entsprechenden Vorstellungen der Anbieter in Form mentaler Karten) in bezug auf Investitionsentscheide. Dabei ging es in erster Linie um die grundsätzliche und in der bisherigen Forschung vernachlässigte Frage, ob räumlich-soziale Kategorien überhaupt eine Rolle für das Handeln von Investoren spielen. Davon ausgehend stellte sich die Frage, wie sich Handlungsweisen der Investoren bei einer Marktliberalisierung in räumlichsozialer Hinsicht verändern würden und mit welchen Folgen zu rechnen wäre.

Die Aktoren lassen sich idealtypisch in die Gruppe der Endinvestoren, die Mittelgruppe und die Promotoren einteilen (HüBsChle et al. 1984, sowie Farago et al. 1993). Die Endinvestoren wurden aufgrund ihrer Heterogenität und ihres grossen Wohnungsbestandes in drei Unterkategorien aufgeteilt: Erstens in die Gruppe der natürlichen Privatpersonen als Vermieterkategorie. Sie wurde über den schweizerischen Hauseigentümerverband angesprochen. Zweitens in die Kategorie der Wohnbaugenossenschaften. Sie waren durch den Schweizerischen Verband für Wohnungswesen vertreten. Und drittens in die Gruppe der institutionellen Anbieter (Pensionskassen, Versicherungen u.a.). Befragt wurde eine grosse Pensionskasse. Die Promotoren wurden über ein grosses Generalunternehmen (GU) angesprochen, die Mittelgruppe, welche in erster Linie die Immobilientreuhänder umfasst, war durch den Schweizerischen Verband der Immobilientreuhänder vertreten. Als Referenzgebiet diente die Stadt Zürich (Abb. 3).

\section{Ergebnisse der Expertenbefragung}

\subsection{Aufschlagspotenziale bei Einführung der Marktmiete}

Der Vertreter der Immobilientreuhänder sieht in heute relativ günstigen citynahen Gebieten (Kreise 4 und 5; vgl. Abb. 1, Abb. 3) bedeutende Aufschlagspotenziale bei Einführung der Marktmiete. Allerdings kommt die Renditesteigerung nicht von selbst; die Strategie heisst «Aufwertung» (Sanierung, Renovation, allgemein: Investitionen). Die Gewissheit, dass diese Strategie Erfolg verspricht und eine kaufkräftige Nachfrage besteht, leitet die befragte Person von ihrer Erfahrung mit einigen Objekten in dieser Gegend (Langstrasse) ab. Die Erfahrung, dass eine Aufwertung möglich und wirtschaftlich sinnvoll ist, wirkt sich auf die Einschätzung des in Zukunft - auch in anderen Grössenordnungen - Machbare aus. Weiter wurde festgehalten, dass im betreffenden Gebiet eine "positive Entwicklung» im Gang sei, die der «Ghettoisierung der letzten zwanzig Jahre», deren Resultat eine «einseitige Zusammensetzung der Wohnbevölkerung» sei, entgegen wirke. Das Aufschlagspotenzial würde sukzessive ausgeschöpft.

Auch die Vertretung des Generalunternehmens sieht im Umfeld der Langstrasse in den Kreisen 4 und 5 Aufschlagspotenziale, weil die Lage zentral ist. Dies gilt aber wiederum nur im Falle einer baulichen Aufwertung. Ohne eine Aufwertung sei kein Aufschlag möglich, weil der «Lärm», die Drogenszene und das Rotlichtmilieu (in der Befragung kurz «Milieu» genannt) höhere Mietpreise verunmöglichten. Die Limitierung der Rendite ist sowohl in der Argumentation des Repräsentanten der Treuhänder als auch derjenigen der GU-Vertretung in erster Linie durch die negativen Folgen der räumlich-sozialen Wohnstandortverteilung gegeben: Ein Preisaufschlag muss von einer Aufwertung begleitet sein, welche die Befragten zwar in erster Linie mit Investitionen verbinden (Sanierungen, «Aufwertungen»), jedoch mit sozialen Veränderungen («sozialer Aufstieg» des Quartiers) begründen. Wanderungsbewegungen werden somit implizit vorausgesetzt, aber nicht expliziert. Der Promotor- und der Treuhändervertreter sind sich also über die Entwicklung im Raum Langstrasse (Kreis 4 und 5) einig.

Die Ansprechperson des GU äusserte sich noch über weitere Quartiere. So seien das restliche Industriequartier (Kreis 5 mit Ausnahme der Langstrasse) und Altstetten (Kreis 9) unattraktive Quartiere, welche nicht begehrt seien und somit auch keine Aufschläge zuliessen. Ebenfalls unattraktiv seien Ober- und Unterstrass (Kreis 6), allerdings wegen qualitiativ unbefriedigenden Wohnungen und nicht wegen der Lage. Anders sei dies in Seebach und Schwamendingen (vgl. Abb. 2). Hier sind die Angebote heute günstig, aber ohne Aufschlagspotenzial, denn das Gebiet sei weder im Grünen, noch am See, noch zentral, sondern industri- 


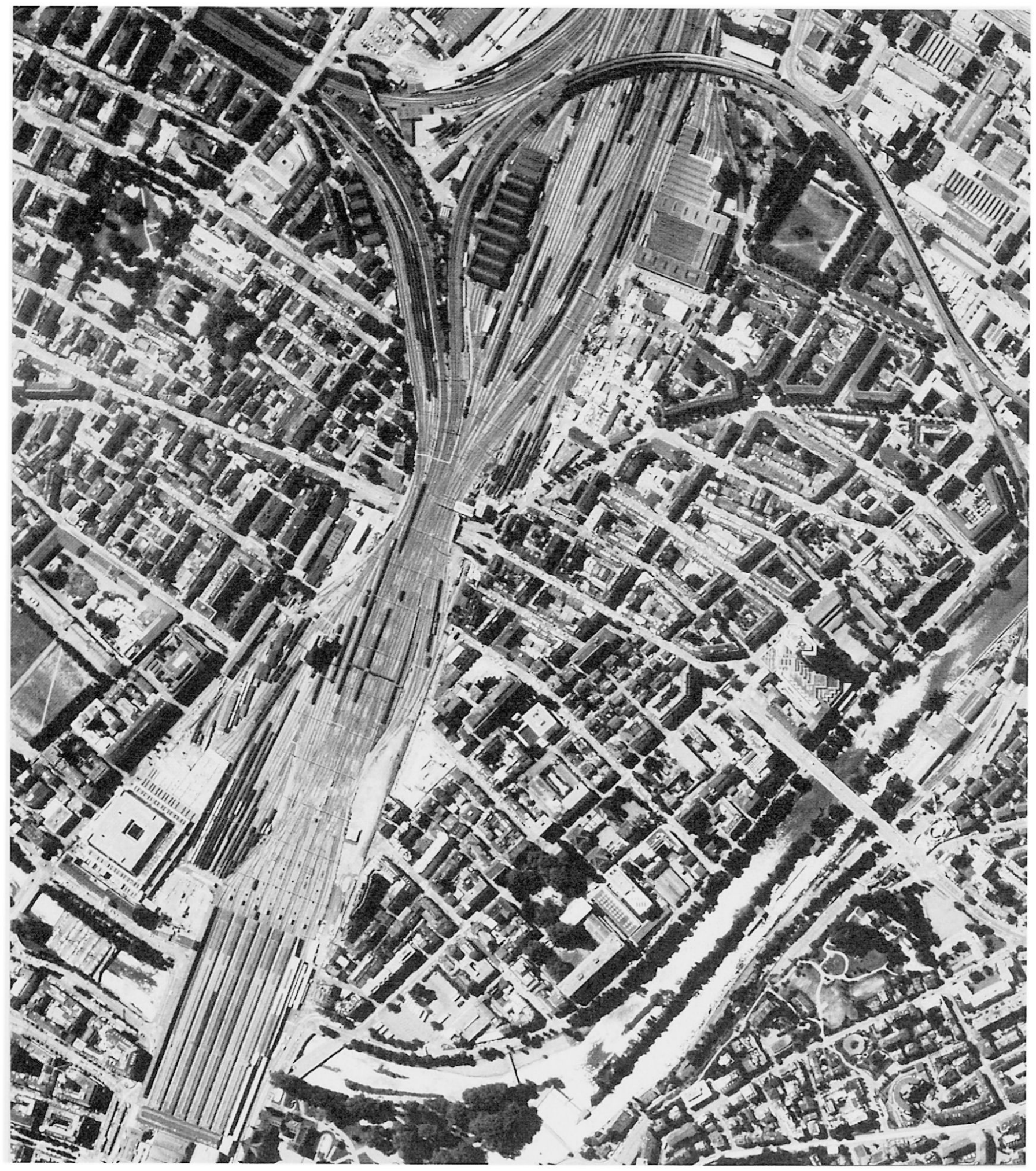

Abb. 1: Zürich: Umgebung der Langstrasse (Kreise 4 und 5)

Zurich: Surroundings of Langstrasse (districts 4 and 5)

Zurich: Les environs de la Langstrasse (arrondissements 4 et 5)

Aufnahme: Bundesamt Für LANDESTOPOGRAPHIE, 11.7.1994, Abdruck mit freundlicher Genehmigung 


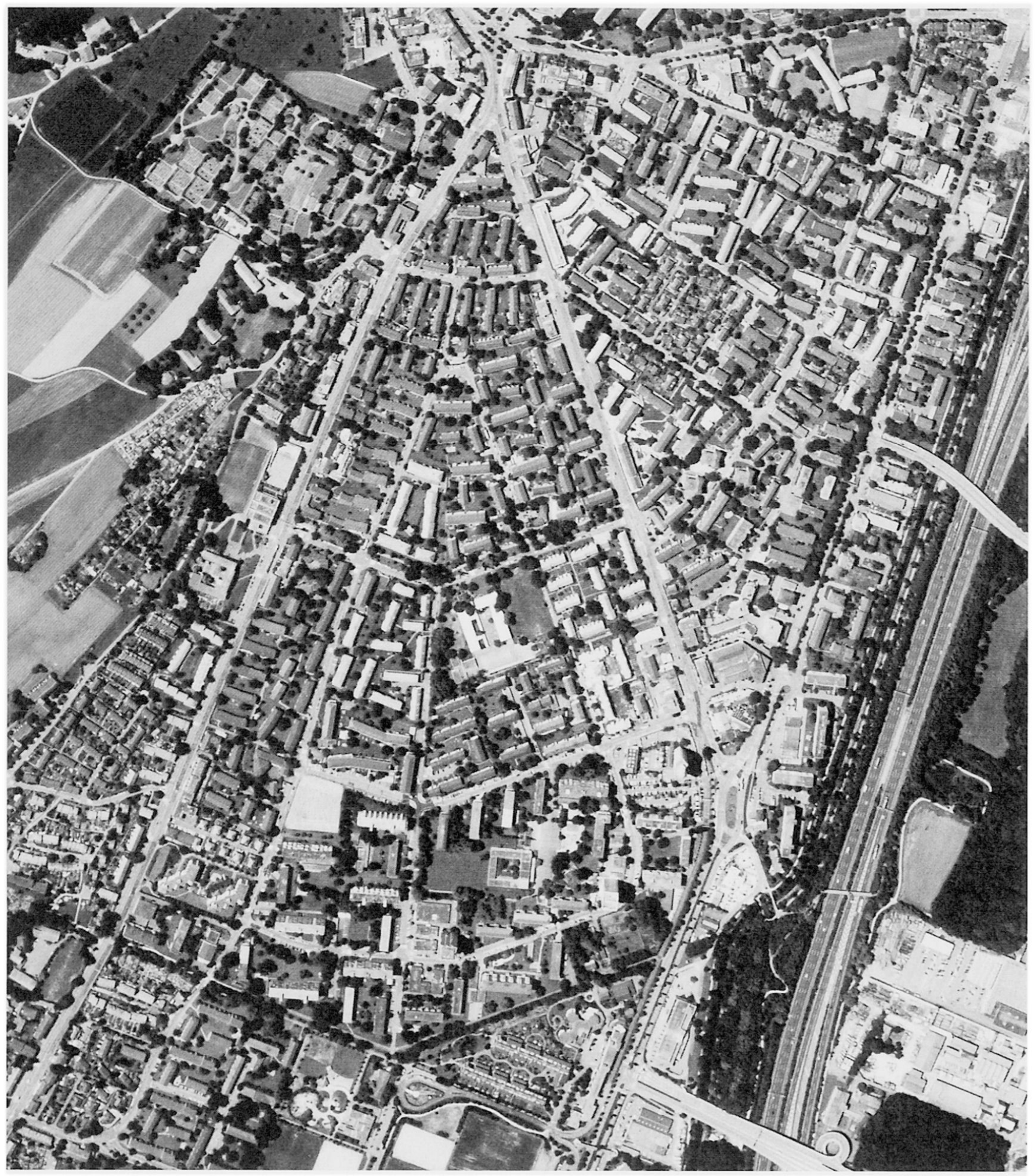

Abb. 2: Zürich: Schwamendingen (Kreis 12).

Zurich: Schwamendingen (district 12)

Zurich: Schwamendingen (arrondissement 12)

Aufnahme: BundeSAmT Für LANDeStopograPhie, 11.7.1994, Abdruck mit freundlicher Genehmigung 
ell geprägt. Hier wolle man nicht wohnen. Das Seefeld hingegen sei heute schon teuer und die Preise schon nahe an Marktpreisen. Aufschläge liessen sich, wenn überhaupt, nur in Altbauten realisieren, welche lange nicht mehr auf dem Wohnungsmarkt angeboten wurden.

Der übereinstimmenden Einschätzung des GU- und des Treuhändervertreters widerspricht die Vertretung der institutionellen Anleger. Nach ihrer Meinung würde der Aufschlag einer Prestigehierarchie folgen: In begehrten Gebieten würden die Investitionen noch zunehmen, in wenig begehrten würden sie eher abnehmen. In heute populären Gebieten sei «nichts zu holen». Die Stadtbevölkerung sei «halt ganz brutal in Kasten unterteilt. Und das wird sich bei einer Liberalisierung auch nicht ändern.» Die Kreise 4 und 5 wären auch nach einer Liberalisierung «nicht plötzlich ein Paradies hoher Renditen", denn in Gebieten, wo die Leute wirtschaftlich schwach seien, würde kaum aufgeschlagen. Als Sozialbarometer diente der befragten Person das Schulwesen. Beispielhaft wurde angeführt, dass jene, die es sich leisten können, aus der Fabrik- und der Josefstrasse (Kreis 5) wegziehen, weil sie für ihre Kinder aufgrund des hohen Ausländeranteils schulische Nachteile befürchten. Die sozial differenzierte Bevölkerungsverteilung scheint für den Pensionskassenrepräsentanten die wesentliche Determinante für die Bewertung eines Quartiers zu sein. Dass sich die «mental maps» auf Handlungsstrategien auswirken, wird deutlich, wenn die Auskunftsperson feststellt: «Wir können ja nicht einfach irgendwo ein bisschen investieren, wenn wir im voraus wissen, es ist schlecht.»

Der Vertreter der Privaten war der Ansicht, dass sich in bezug auf die Aufschlagsmöglichkeiten bei einer Marktmiete-Einführung gar nichts ändern würde und wollte sich deshalb auf keinerlei Spekulationen einlassen. Der Markt sei übersättigt und deshalb sei die Frage nach Preissteigerungen «out». Er ist der Ansicht, dass es nur in wirklichen Top- oder Prestigelagen mit einem anderen Mietrecht Aufschläge geben könne. Er fügte ironisch hinzu: "Also wenn einer in Zollikon wohnen will, dann ist es nicht das Mietrecht, das ihn daran hindert...».

Ganz im Gegensatz zu den renditeorientierten Anbietern würden die Wohnbaugenossenschaften mögliche Aufschlagspotenziale nicht ausschöpfen. Die Renditemaximierung stellt kein vorrangiges Ziel dieser Anbietergruppe dar. Vielmehr bezeichnete der Vertreter der Wohnbaugenossenschaften die Schaffung bzw. den Erhalt von sozial ausgeglichenen Quartieren als ein eigentliches Hauptziel der Aktivitäten der gemeinnützigen Genossenschaften. Auch er nimmt die Schulen als Gradmesser für die Durchmischung. Die räumlich-soziale Wohnstandortverteilung stellt eine wichtige Orientierungsgrösse dar, welche intensiv beobachtet wird. Schliesslich bildet die soziale Durchmischung in genossenschaftlich dominierten Quar- tieren eine Art Erfolgskontrolle für die Politik der Wohnbaugenossenschaften.

Zusammenfassend kann festgehalten werden: Alle Aktoren verfügen über klare Vorstellungen bezüglich der sozialen und baulichen Prägung der Quartiere. Sie sind sich einig über die Ausgangssituation, d.h. über die räumlich-soziale Prägung von Quartieren, nicht aber über die zukünftige Entwicklung, wie die Diskussion der Kreise 4 und 5 zeigt. In den Gesprächen wurde auch deutlich, dass sämtliche Befragten ihrer mentalen Kartierung der räumlich-sozialen Wohnstandortverteilung ein grosses Gewicht im Rahmen der Beurteilung von Investitionsentscheiden beimessen. Die Einschätzung der Qualität von Quartieren durch die Investoren erfolgt ähnlich wie bei der besser bekannten Einschätzung der Mieter durch die Vermieter (AREND et al. 1990) aufgrund von zugeschriebenen Eigenschaften in Form einzelner Attribute. Es wird bei der Beurteilung von Wohngebieten von Teilaspekten auf die Qualität ganzer Quartiere geschlossen. Als Beispiele gelten die in den Expertengesprächen genannten Attribute wie der Ausländeranteil, gemessen an der «Schulbelastung» oder die Vorherrschaft bestimmter Gruppen, angesprochen mittels «einseitiger Zusammensetzung der Wohnbevölkerung» und «Milieu», als Indikatoren für die Quartierqualität.

Die bisherigen Ergebnisse werden im Folgenden mit der Frage verknüpft, wie sich Investitionsstrategien veränderten, wenn die Marktmiete eingeführt würde. Die Investitionsstrategien wurden in den Gesprächen mittels der Gewichtung verschiedener Ziele innerhalb des Wohnungswesens erfragt. Ausgehend von Kritikpunkten am geltenden Recht kann hypothetisch geschlossen werden, welche veränderten Handlungsweisen eine Liberalisierung des Rechts nach sich ziehen würde.

\subsection{Kritikpunkte am geltenden Mietrecht}

Alle Befragten bemängelten an der heutigen Regelung, dass Rücklagen für Unterhaltsarbeiten behindert werden. Die treuhänderisch Tätigen forderten eine Risikoprämie, welche dem Ausgleich von Leerständen dienen soll. Weiter wurde betont, dass das Mietrecht einen Ausgleich von Verlusten durch Gewinne verunmögliche. Jene, die mit einem grossen Anteil Eigenkapital arbeiten (v.a. institutionelle Anbieter), bemängeln die im Mietrecht unterstellte Kapitalaufteilung (40\% Eigenkapital zu 60\% Fremdkapital), welche eine vollständige Überwälzung wertvermehrender Investitionen auf die Mieter verbietet. Die Preisschere zwischen Alt- und Neuwohnungen wurde von allen renditeorientierten Aktoren bemängelt. Viele Wohnungen im Altbestand seien heute zu billig. Unbestritten ist, dass die Wohnungsmieten umso höher sind, je jünger das Baudatum ist. Allerdings stimmt dies nicht in genereller Form: Die Wohnungen aus der Zeit 1947-1960 sind billiger als jene aus den vorangegan- 
genen Epochen. Und kürzlich renovierte Altbauten (Baujahr vor 1947) sind erheblich teurer als neuere Wohnungen, die ebenfalls renoviert wurden (NORTH 1996: 52). Mit grösser werdendem Bestand renovierter Wohnungen verliert die «Preisscheren-Regel» zunehmend an Bedeutung.

In bezug auf die Anwendbarkeit der Orts- und Quartierüblichkeit (=Vergleichsmiete: Anhand einer Auswahl von Wohnungen oder Geschäftsräumen, die einander hinsichtlich Lage, Grösse, Ausstattung, Zustand und Bauperiode entsprechen, ermittelter Mietpreis) sind sich Treuhänder-, GU- und Pensionskassenvertreter einig, dass dieses Marktelement aufgrund der Bundesgerichtspraxis kaum mehr anwendbar sei. Dies wird als ein grosses Hindernis in bezug auf die Praxis der Mietpreisgestaltung verstanden.

\subsection{Neue Investitionsstrategien im veränderten rechtlichen Umfeld}

Eine Kernaussage der Interviews ist, dass sich die Aktoren nach einer Marktliberalisierung - wie sie dies heute schon tun - an der kaufkräftigen Nachfrage orientieren. Im Unterschied zu heute würden aber keine Verfahrenssituationen antizipiert, was die Mietpreise in der Tendenz steigen lässt. Dies deshalb, weil bei einer Deregulierung des Mietrechts, welches Regelungen betreffend der Mietpreise beinhaltet, nicht aus Angst vor Verfahren auf Aufschläge verzichtet würde, wie dies heute geschieht. Bei Renovationen wird einerseits im Rahmen des am Markt Möglichen stärker aufgeschlagen, was vor allem im Interesse jener Investoren liegt, die mit einem grossen Anteil Eigenkapital arbeiten (v.a. institutionelle Anbieter). Andererseits werden Kündigungen vereinfacht, was Totalsanierungen begünstigt. Die angesprochene stärkere Gewichtung der Quartierüblichkeit, welche der marktmässigen Bewertung einer Umgebung entspricht, leistet einer Homogenisierung der Bevölkerung nach ökonomischen Gesichtspunkten innerhalb der Quartiere tendenziell Vorschub.

In bezug auf die Lesart der Quartiere in räumlichsozialer Hinsicht unterscheiden sich die Aktoren nicht wesentlich. Was hingegen die Interpretation der räumlich-sozialen Ausgangslage betrifft, so scheinen sich verschiedenartige Handlungsweisen herauszubilden. Obwohl verschiedene Investoren die räumlichsoziale Prägung von Quartieren gleich lesen, gelangen sie zu unterschiedlichen und mitunter gegensätzlichen Einschätzungen der entsprechenden Entwicklungspotenziale. Besonders deutlich wurde dies in bezug auf die innerstädtischen Quartiere der Kreise 4 und 5.Während die Interpretation des Pensionskassenrepräsentanten keine Zweifel daran lässt, dass er eine Verstärkung der bestehenden räumlich-sozialen Disparitäten für wahrscheinlich hält, weist die Strategie des Treuhänderund des GU-Vertreters in eine andere Richtung. Eine wichtige Voraussetzung für Segregationsprozesse ist hiermit gegeben. Die Rolle der residenziellen Segregation bzw. ihrer Perzeption im Sinne mentaler Karten besteht darin, dass mittels der räumlich-sozialen Wohnstandortverteilung die Entwicklungspotenziale eines Quartiers abgeschätzt werden. Somit erfolgt eine erste Einschätzung, welche Investitionsweise (z.B. AbbruchNeubau, Totalsanierung, «Pinselrenovation», gar keine Renovationen etc.) im entsprechenden Quartier sinnvoll ist.

In bezug auf veränderte Investitionsstrategien wurden zwei Optionen pointiert geäussert. Es sind dies einerseits eine Aufwertungsstrategie der risikobereiteren Investoren und auf der anderen Seite eine konservative Strategie.

Die Aufwertungsstrategie führt zu einer Zunahme von Investitionen in innenstadtnahen Gebieten, die im Zuge der Suburbanisation zu typischen Beispielen für A-Quartiere (Arme, Asylsuchende, Auszubildende, Aussteiger, Ausländer, Alte...) geworden sind (FrEY 1996: 15). Der Wunsch der renditeorientierten Vertreter der Immobilieninvestoren nach neuen Verwertungsmöglichkeiten geht gemäss KRÄTKE (1995: 177) einher mit dem Wunsch zahlungskräftiger Gruppen, in zentral und verkehrsgünstig gelegenen urbanen Quartieren zu wohnen. Eine unbeabsichtigte räumlichsozial relevante Folge dieser von Nachfrage- und Anbieterseite gemeinsam konstituierten Entwicklung sind Verdrängungsprozesse.

Die konservative Strategie findet Anwendung bei risikoaversen Anbietern, welche ihre Anlagestrategie aufgrund der veränderten rechtlichen Rahmenbedingungen dennoch aktiv überprüfen. Sie investieren entlang einer Prestigehierarchie, wovon Gebiete profitieren, welche heute schon begehrt und (auch von der baulichen Substanz her) attraktiv sind, in denen eine genügende Nachfrage besteht, und die Bevölkerung von heute zumindest durchschnittlich zahlungskräftig ist. Dies lässt erwarten, dass die Überwälzung der Mehrinvestitionen via Mietpreiserhöhungen in diesen Gebieten von den dortigen Bewohnern getragen werden könnte. Die Bevölkerungszusammensetzung würde sich folglich nicht wesentlich verändern. Die Attraktivität entsprechender Quartiere äussert sich heute in Form eines Nachfrageüberhangs, was die Einschätzung, wonach sich die Investition auszahlen wird, stützt.

Die aufwertende und die konservative Strategie decken sich in bezug auf den Umgang mit peripheren Gebieten. Diese Gebiete kommen weder in die Gunst von Investitionen, die einer Prestigehierarchie folgen, noch in solche, welche von der Zentralität her heute unterbewertete Gebiete speisen. So wären periphere Gebiete mit einer ökonomisch schwachen Bevölkerung die Verlierer des Prozesses. Die betreffenden Quartiere würden baulich abgewertet bzw. nur minimal unterhalten, was eine Ballung von sozial schwächeren Haushalten mit geringer Mobilität tendenziell verstärkt. 


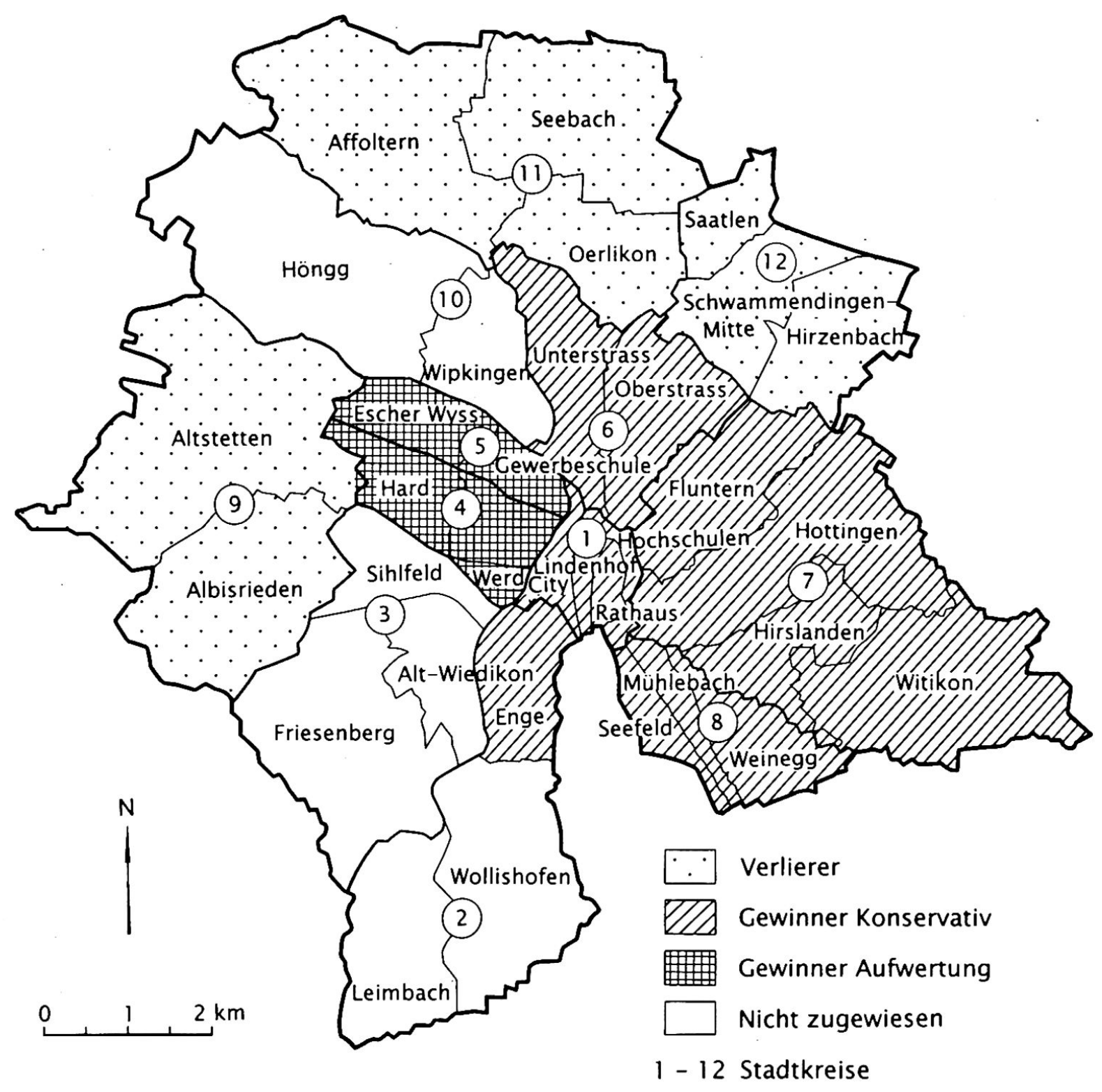

Abb. 3: Auf- und Abwertungsquartiere der Stadt Zürich

Residential areas with real estate revaluation or devaluation tendencies in the City of Zurich Quartiers présentant des tendences de dévalorisation ou revalorisation dans la ville de Zurich Quelle: VAN WeZEmael 1999: 171; Kartengrundlage: Strukturatlas der Schweiz 1997; Bearbeitung: L. BaumanN

Die genannte Entwicklung fördert so die Polarisierung der Quartiere und deckt sich mit der zunehmenden Hierarchisierung von Konsummustern auf der Nachfrageseite. Der Entstehung von Armutsinseln sowie aufgewerteten Gentrifizierungsquartieren würde durch die Einführung der Marktmiete Vorschub geleistet.

\subsection{Räumlich differenzierte Anwendung der Strate- gien am Beispiel der Stadt Zürich}

Mittels einer sekundärstatistischen Auswertung von Daten des Statistischen Jahrbuchs von Zürich (1997/1998) wurden die Stadtzürcher Quartiere charakterisiert und anschliessend diejenigen Gebiete gesucht, welche die in den Expertengesprächen genannten Voraussetzungen für die Anwendung der aufwertenden oder der konservativen Strategie erfüllen. Quartiere, welche über entsprechende Voraussetzungen verfügen, wurden als Gewinner bezeichnet. Die Absenz der Voraussetzungen für beide Strategien führte zur Ausscheidung der Verliererquartiere. Quartiere, die in bezug auf die Investitionsstrategien nicht eindeutig charakterisierbar waren, wurden nicht zugewiesen. Mit Gewinner ist gemeint, dass in die entsprechenden Quartiere im Ver- 
gleich zur heutigen Situation mehr investiert wird und dass diese Quartiere in der relativen sozialen Rangskala der Stadtquartiere tendenziell steigen. Für Verlierer gilt entsprechend das Umgekehrte. Gewinner und Verlierer sind als wertneutrale Bezeichnungen zu verstehen. Dass es gerade in den aufgewerteten Gewinnerquartieren «Verliererschicksale» geben kann, soll mit der pragmatischen Begriffsdefinition nicht geleugnet werden. Abb. 3 fasst das Ergebnis des Szenarios anschaulich zusammen.

Zu den Gewinnerquartieren der Aufwertungsstrategie gehören die Kreise 4 und 5, die sich durch «urbanes Flair», die zentrale Lage sowie eine gründerzeitlich dichte Bebauung auszeichnen. Die Mietpreise liegen relativ tief und die Bewohnerstrukturen zeichnen sich heute durch Bewohner tieferer sozio-professioneller Kategorien sowie einen hohen Ausländeranteil aus. Verdrängungsprozesse im Gefolge der Aufwertung werden die jetzigen Bewohner betreffen.

$\mathrm{Zu}$ den Gewinnerquartieren der konservativen Strategie zählen die Kreise 1, 6, 7 und 8 sowie das Teilgebiet Enge des Kreises 2. Dies sind bereits heute bevorzugte und begehrte Wohnlagen am Zürichberg und in Seenähe, die eine lockere bis dichte Bebauung gehobeneren Standards aufweisen. Die Mieten sind im Vergleich zum städtischen Durchschnitt überdurchschnittlich. Die Bewohnerstrukturen lassen sich durch einen grossen Anteil von Bewohnern höherer sozio-professioneller Kategorien und einen vergleichsweise tiefen Ausländeranteil charakterisieren. Aufgrund der konservativen Aufwertungsstrategie werden sich die bestehenden Strukturen festigen und Verdrängungsprozesse sind wenig wahrscheinlich.

$\mathrm{Zu}$ den Verliererquartieren zählen die Kreise 11 und 12 (vgl. Abb. 2) im Norden der Stadt Zürich sowie der Kreis 9 im Westen. Sie sind tendenziell wenig begehrt, was u. a. auf die periphere Lage, das negative Image und die hohe Lärm- und Luftbelastung zurückzuführen ist. Dieser Umstand drückt sich auch in einer vergleichsweise hohen Leerstandsquote aus. Weiter weisen diese Quartiere bedeutende Anteile genossenschaftlicher Wohnungen auf, was zu hohen Anteilen von Familien, aber auch von sozioökonomisch eher schwächeren Haushalten führt. Ohne Aufwertungsstrategien der öffentlichen Hand und der Genossenschaften dürften sich in gewissen Teilen dieser Kreise Desinvestitionsprozesse und eine Verschlechterung der Quartierstrukturen bemerkbar machen.

\section{Fazit}

Die Befragungsergebnisse belegen die Wichtigkeit der räumlich-sozialen Dimension als Orientierungsgrösse für das Handeln der Investoren innerhalb der Woh- nungswirtschaft. Die Analyse der Bezugnahme auf die räumlich-soziale Dimension als Handlungsbedingung ist somit eine grundlegende Voraussetzung zum Verständnis des Geographie-machens der Aktoren im Rahmen ihres alltäglichen Handelns. Die räumlichsoziale Wohnstandortverteilung ist sowohl als Kernelement in der Handlungsorientierung der Investoren im Wohnungsimmobilienmarkt als auch in bezug auf die Reproduktion der residenziellen Segregation zu verstehen. Bei Einführung der Marktmiete ist mit einer Modifikation der Prozesse der Reproduktion der residenziellen Segregation zu rechnen. So würden mittels einer Zunahme der Ungleichheit der Investitionsströme zwischen verschiedenen Typen von Quartieren sowohl neue Disparitäten geschaffen wie bestehende verstärkt werden. Das Räumliche darf folglich nicht länger aus der Diskussion um die Ausformung der Marktsphäre im Mietwesen ausgeschlossen bleiben.

\section{Literatur}

Arend, M., Kellerhans Spitz, A. \& T. Mächler (1990): Benachteiligte Gruppen auf dem Wohnungsmarkt. Probleme und Massnahmen. - = Schriftenreihe Wohnungswesen 45, Bern: Bundesamt für Wohnungswesen.

B̈̈RTSCHI, H.P. (1983): Industrialisierung, Eisenbahnschlachten und Städtebau. - In: Schriftenreihe des Instituts für Geschichte und Theorie der Architektur an der ETH 5, Basel: Birkhäuser.

BORNSCHIER, V.(1998):Westliche Gesellschaft - Aufbau und Wandel. - Zürich: Seismo.

Bourdieu, P. (1991): Physischer, sozialer und angeeigneter physischer Raum. - In: WENTZ, M. (Hrsg.): Stadt - Räume. - Frankfurt am Main: Campus.

Bundesamt Für Wohnungswesen (1993) (Hrsg.): Bericht der Studienkommission Marktmiete. - = Arbeitsberichte Wohnungswesen 28, Bern: Bundesamt für Wohnungswesen.

De Pury, D., Hauser, H. \& B. Schmid (Hrsg.) (1995): Mut zum Aufbruch. Eine wirtschaftspolitische Agenda für die Schweiz. - Zürich: Orell Füssli.

Farago, P., Hager, A. \& C. Panchaud (1993): Verhalten der Investoren auf dem Wohnungsimmobilienmarkt. - = Schriftenreihe Wohnungswesen 54, Bern: Bundesamt für Wohnungswesen.

FDP (2000):PositionspapierWohnen.-http://www.fdp.ch/ FDP/POS_PAP/wohnen.htm, 14.06.2000.

FLICK, U. (1998): Qualitative Forschung. Theorie, Methoden, Anwendung in Psychologie und Sozialwissenschaften. - 3. Auflage, Reinbek bei Hamburg: Rowohlt-Taschenbuch.

FREY, R.L. (1996): Stadt: Lebens- und Wirtschaftsraum. Eine ökonomische Analyse. - Zürich: vdf Hochschulverlag.

GidDENS, A. (1995): Die Konstitution der Gesellschaft: Grundzüge einer Theorie der Strukturierung. - Frankfurt am Main und New York: Campus. 
HaRTKE, W. (1959): Gedanken über die Bestimmung von Räumen gleichen sozialgeographischen Verhaltens. - In: Erdkunde 13/4: 426-436.

Hübschle, J., Herbst, M. \& K. Eckerle (1984): Investorenverhalten auf dem schweizerischen Wohnungsmarkt. Unter besonderer Berücksichtigung gruppenund regionsspezifischer Merkmale. - = Schriftenreihe Wohnungswesen 31, Bern: Bundesamt für Wohnungswesen.

JÄGER, F. \& S. BÜHLER (1995): Marktmiete. Schweizer Wohnungsmieten zwischen Politik und Markt. - Chur und Zürich: Rüegger.

KRÄTKE, S. (1995): Stadt, Raum, Ökonomie. Einführung in aktuelle Problemfelder der Stadtökonomie und Wirtschaftsgeographie. - = Stadtforschung aktuell 53, Basel, Berlin und Boston: Birkhäuser.

LiChTENBERGER, E. (1998): Stadtgeographie 1:Begriffe, Konzepte, Modelle, Prozesse. - Stuttgart und Leipzig: Teubner.

NoRTH, Y. (1996): Der Gebäudebestand: Seine Zusammensetzung und Entwicklung. - = Wohnen in der Schweiz. Fachbereich 9: Bau- und Wohnungswesen. Statistik der Schweiz, Bern: Bundesamt für Statistik. Odermatr, A. (1997): Eigentümerstrukturen des Wohnungsmarktes. Ein handlungstheoretischer Beitrag zur Erklärung der räumlich-sozialen Wohnstandortverteilung am Fallbeispiel der Schweiz. - Münster: LrT Verlag. SCHIPS, B. \& E. Müller (1993): Mietzinsniveau bei Marktmieten. - In: BUNDESAMT FÜR WOHNUNGSWESEN (Hrsg.): Materialien zum Bericht der Studienkommission Marktmiete. - Arbeitsberichte Wohnungswesen 29, Bern: Bundesamt für Wohnungswesen.

Smelser, N. J. \& R. Swedberg (1994): The Sociological Perspective on the Economy. - In: SMelser, N.J. \& R. Swedberg (Hrsg.): The Handbook of Economic Sociology. - Princeton: Princeton University Press: 3-26.

Statistisches Amt der Stadt Zürich (Hrsg.) (1997): Statistisches Jahrbuch 1997, 92. Jg. - Zürich: Statistisches Amt.

Statistisches Amt der Stadt ZüRICH (Hrsg.) (1998): Statistisches Jahrbuch 1998, 93. Jg. - Zürich: Statistisches Amt.

Van Wezemael, J. E. (1999): Markt und Wohnen. Ein Beitrag zur Marktmiete-Debatte aus sozial- und wirtschaftsgeographischer Sicht. Diplomarbeit am Geographischen Institut der Universität Zürich. - Zürich. WERLEN, B. (1997): Sozialgeographie alltäglicher Regionalisierungen, Band 2: Globalisierung, Region und Regionalisierung. - Stuttgart: Franz Steiner Verlag.

\section{Zusammenfassung: Verändert die Marktmiete die residenzielle Segregation? Die Marktmiete aus sozial- und wirtschaftsgeographischer Sicht}

Im vorliegenden Artikel wird die im Kontext der Deregulierungsdebatte diskutierte Folge des Systemwechsels hin zur Marktmiete für Wohnungen aus einer sozial- und wirtschaftsgeographischen Perspektive ausgeleuchtet. Hierzu wurden die möglichen Änderungen auf die Investitionsstrategien der Anbieterseite des Wohnungsmarktes analysiert. Aufgrund der Expertenbefragung zeigte sich, dass die bisher vernachlässigte räumlich-soziale Dimension eine bedeutende Orientierungsgrösse der Investitionshandlung darstellt. Weiter konnten zwei idealtypische Investitionsstrategien unterschieden werden. Die konservative Strategie würde nach Einführung der Marktmiete zu vermehrten Investitionen in bereits begehrten Quartieren führen, während die Aufwertungsstrategie Gentrifizierungsprozesse in innenstadtnahen Quartieren auslösen oder fördern würde. Neben diesen beiden Gebietstypen, die ein Aufschlagspotenzial bei der Einführung der Marktmiete aufweisen, gibt es auch Quartiere, in welchen die Investitionstätigkeit eher abnehmen würde, was zu Abwertungstendenzen führte. Bei der Einführung der Marktmiete würde sich somit die räumliche Homogenisierung der Bevölkerung nach ökonomischen Kriterien und die räumlich-soziale Polarisierung der Siedlungsstrukturen verstärken.

\section{Résumé: Est-ce que le loyer de marché aura des conséquences sur la ségrégation résidentielle?} Le loyer de marché du point de vue de la géographie socio-économique

L'article présent examine du point de vue de la géographie socio-économique les conséquences d'un changement de système en faveur d'un loyer de marché du logement, changement qui fait l'objet du débat sur la dérégularisation. A cet effet, on a analysé les changements possibles sur les stratégies d'investissement du côté des offres du marché du logement. Un sondage auprès des experts a montré que la dimension spatio-sociale, négligée jusqu'à présent, représente un facteur d'orientation important pour l'activité d'investissement. De plus, on est arrivé à différencier deux stratégies d'investissement typiques dans le sens idéal du terme. La stratégie conservative engendrerait, après l'introduction du loyer de marché, des investissements accrus dans les quartiers en vogue, tandis que la stratégie de revalorisation déclencherait ou encouragerait des processus de gentrification dans les quartiers proches du centre-ville. En plus de ces deux types de quartier qui représentent un potentiel de renchérissement lors d'une éventuelle introduction du loyer de marché, il existe aussi des quartiers dans lesquels l'activité d'investissement aurait plutôt tendance à reculer, ce qui mènerait à des processus de dévalorisation. Selon les critères économiques, l'introduction du loyer de marché aurait donc pour conséquence une accentuation de l'homogénisation spatiale de la population ainsi qu'une polarisation spatio-sociale des structures de l'habitat. 


\section{Summary: Does Decontrolled Rent Affect Residen- tial Segregation? Decontrolled Rent from a Socio- and Economic-Geographical Perspective}

Within the context of the deregulation debate, the present article illuminates the repercussions of introducing decontrolled rents in the housing market. Possible changes in the investment strategies of suppliers in the housing market are analyzed. A survey of experts reveals that spatio-social dimensions represent a significant orientation for investment. Two investment strategies are distinguished. After introducing decontrolled rents, the conservative strategy may lead to increased investments in already attractive residential areas. The revaluation strategy, on the other hand may trigger or promote gentrification in residential areas bordering on the inner city. In addition to areas that may benefit from the introduction of decontrolled rents, there are residential areas in which investments would tend to decrease, leading to devaluation. Consequently, the introduction of decontrolled rents may intensify both the spatial homogenization of the population according to economical criteria and the spatiosocial polarization of residential areas.

Dipl. phil. Joris Ernest Van Wezemael, Dr. phil. André Odermatt, Geographisches Institut, Universität Zürich, Abteilung Wirtschaftsgeographie, Winterthurerstrasse 190, CH-8057 Zürich.

e-Mail:

wezemael@geo.unizh.ch

odermatt@geo.unizh.ch 